\title{
Exploring the Requirements and Design of Persuasive Intervention Technology to Combat Digital Addiction
}

\author{
Amen Alrobai, John McAlaney, Huseyin Dogan, Keith Phalp, Raian Ali \\ Faculty of Science and Technology \\ Bournemouth University, United Kingdom \\ \{aalrobai, jmcalaney, hdogan, kphalp,rali\}@bournemouth.ac.uk
}

\begin{abstract}
Digital Addiction (DA) is an emerging behavioural phenomenon that denotes an obsessive and problematic usage of digital media. Such usage could meet various criteria of an addictive behaviour such as salience, conflict, tolerance and withdrawal symptoms and, hence, it would raise new challenges and ethical considerations on the way we engineer software. Luckily, software as a medium for such addictive usage could be also a medium for enacting a behaviour change and prevention strategy towards a regulated usage. However, due to the recentness of such software-based interventions, we still need a body of knowledge on how to develop them. In this paper, we conduct empirical research, through a diary study and an online forum content analysis, to understand users' perception of such emerging systems. The results shed the light on a range of design aspects and risks when building and validating such persuasive intervention technology.
\end{abstract}

Keywords: Digital Addiction, e-Heath design, design for behavioural change.

\section{Introduction}

The wealth of information and digital connectivity is a characteristic of a modern society but its excessive and obsessive use may result in a less sustainable society and create social and mental well-being problems. The consequences of such Digital Addiction (hereafter DA) on individuals and collectively, include poor academic performance, reduced social and recreational activities, relationships breakups, low involvement in real-life communities, poor parenting, depression and lack of sleep $[1,2,3]$. DA manifests psychological characteristics and along with dependency, the user can experience withdrawal symptoms (e.g. depression, cravings, insomnia, and irritability). Estimates of DA vary according to country and according to the definition of DA and the metrics used to measure it in the studies. Such estimates of internet addiction suggest that $6 \%-15 \%$ of the general population test positive on signs of addiction; this figure rises to $13-18 \%$ among university students who have been identified as most at risk for DA [4]; at $18.3 \%$ UK has a relatively high prevalence of DA amongst university students [5].

The existing literature on DA has focused mainly on users' psychology. There is a paucity of research that positions software and its developers as primary actors in the 
development of DA. A notable exceptions are the research in [6,7] which advocates that by developing DA-aware architecture and design, software developers can minimise addictive usage and thereby prevent or intervene early with DA. Software developers, together with users, can use inputs from technology and psychology to create DA-aware software and facilitate a healthy use of digital technology.

Technology-assisted behaviour change is an emerging topic and we are witnessing an increase in its adoption in several domains and for different addictive and problematic behaviours. For example, online intervention is being used for alcohol addiction and encouraging a responsible drinking [8]. Also, the advances in information technology and Web 2.0 have enabled a new range of possibilities including a more intelligent, context-aware, continuous and social online intervention. As evidence, the use of mobile applications for behaviour change is now a possibility, e.g. for smoking cessation [9], medication adherence [10], diet and eating disorder [11], to name a few.

Despite the trend, there are still few principles and design guidelines on how technology-assisted behaviour change should be engineered. Amongst other aspects, we lack studies on users' views and their requirements, personal and collective [12]. In general, there is a limited amount of theory-based solutions and this deters their acceptance, efficiency, usability and sustainability. In developing such solutions, there seem to be interesting intersections amongst several disciplines. For example, topics like personalisation, either based on automatic adaptation or user's direct modifications [13], social norms and social comparisons [8] which fall within a psychology remit, would be familiar concepts in computing areas such as requirements personalisation [14] and persuasive technology [15].

Software design can play a key role in facilitating addictive behaviours. Certain interactivity can trigger preoccupation and an escalation of commitment and tendency to allocate additional time and to a chosen task, e.g. in forum or email conversation. Other can trigger the fear of missing out events that maybe currently happening, e.g. newsfeeds in a social network. At the same time, we argue that software enjoys capabilities that can offer breakthrough solutions to manage such addictive behaviour. This includes being transparent to users and providing real-time traceability of their usage and intelligent and personalized feedback messages. Unlike other addictive mediums, e.g. tobacco and alcohol, software can aid users to take an informed decision of their usage more actively.

In this paper, we study a set of commercial e-health persuasive applications to combat DA and collect evidence of their capabilities, design defects and their potential to cause adverse impact. We explore such persuasive intervention technology (hereafter PIT) from their users' perspective. This will inform software engineering about the relevant requirements and design facets and concerns and paradoxes to cater for. We follow a qualitative approach and analyse users' online feedback on a set of popular PIT and conduct a diary study with a group of users having a problematic usage style to capture their experience with such technology for a period of time. We conclude with a set of recommendations to follow and risks to avoid when designing PIT for combatting DA.

The paper is structured as follows. We first present our method in Section 2. Then we present the results in four categories of features related to PIT in Section 3. We 
then reflect on the results from both design and psychological perspectives with the aim to inform their development in Section 4. Study limitations presented in section 5. Finally, we draw conclusion and presents our future work in Section 6.

\section{Method}

We followed a qualitative method to understand users' perception of PIT for combatting DA. Overall, multiple data sources were used to increase coverage and credibility of the study. The first was the diary study to collect data in naturalistic settings. The second was the follow-up individual interviews to develop better understanding of the data collected from the diary studies. The third was the analysis of an online forum to gather more contextual knowledge about these applications. For an exploratory study, we treated the data coming from the three sources equally and made the content analysis under the assumption that such diversity will reveal more concepts.

We began with reverse engineering three popular smartphone application designed to aid users regulate their usage and reduce their DA to extract their notable features. An extra application (App.4) has been included later in the study. The reason will be discussed in the following paragraph. Table 1 outlines the features of these applications and categorises them based on their support dimension [16]. The popularity was measured through the number of installs (over 1M) and feedback provided (over 5K). We then aimed to get users' perception of these features. This helped to decide the prominent and significant features and to look at the requirements and contextual factors that can influence their effectiveness and deficiencies.

Table 1. The features and design principles of the selected applications

\begin{tabular}{|c|c|c|c|c|c|}
\hline & Features & App.1 & App. 2 & App.3 & App.4 \\
\hline \multirow{4}{*}{ Task support } & Monitoring \& tracking & $\bullet$ & $\bullet$ & $\bullet$ & $\bullet$ \\
\hline & Coercive techniques & $\bullet$ & $\bullet$ & $\bullet$ & $\bullet$ \\
\hline & Goal settings & $\bullet$ & $\bullet$ & $\bullet$ & \\
\hline & Tunnelling & & & & \\
\hline \multirow{5}{*}{ Social support } & Competition & & $\bullet$ & & $\bullet$ \\
\hline & Normative influence & & & $\bullet$ & \\
\hline & Recognition & & & $\bullet$ & \\
\hline & Social support & & $\bullet$ & $\bullet$ & $\bullet$ \\
\hline & Comparisons & $\bullet$ & & & \\
\hline \multirow{3}{*}{ Dialogue support } & Rewarding & $\bullet$ & $\bullet$ & $\bullet$ & $\bullet$ \\
\hline & Reminders & $\bullet$ & $\bullet$ & $\bullet$ & \\
\hline & Addiction scoring & & $\bullet$ & $\bullet$ & \\
\hline
\end{tabular}

In the diary study, 14 participants were recruited ( 5 females and 9 males, with ages ranging between 18 and 50). They were asked to use the three commercial PIT to combat DA for two weeks and write down their observations and feelings about them and their usage style. They were also asked to take snapshots of significant moments during the usage and share that with the research team at least once every two days. The data gathered was then used to support our interviews with those participants after the two weeks. The interviews were audio recorded and transcribed. The studies 
followed the principles and guidelines presented in [17]. The recruitment was based on convenience sampling. The inclusion criterion was that the participants should have the feeling that the smartphones or social media is used in an excessive and obsessive way. Participants who met that criterion were then sent an invitation email with a short questionnaire to complete.

The research indicates that self-reports in which participants are simply asked if they thought they have DA are strongly correlated with available psychometric measures to assess DA [18]. However, CAGE questionnaire [19] was also used as a self-assessment instrument for further validity check. The participants all declared at least one aspect of problematic usage of their smartphones; they passed a preselection survey test which was designed based on the CAGE questionnaire which is a screening self-report instrument to detect addictive behaviours by examining the addiction symptoms such as conflict, tolerance, withdrawal symptoms, mood modification and salience.

The other data source utilised users' online feedback and review on the same three applications. However, we noticed from the analysis of the diary study data that users wanted to be motivated by some sort of rewarding systems that reinforce their sense of accomplishments and care of some virtual object of character. As such, we analysed one more application that represents users' achievements metaphorically by providing them with virtual experience of looking after something, e.g. a tree or a pet, which would become less healthy or less happy when they are busy with their usage of digital technology.

In the analysis of users' online reviews and feedback, we analysed 733 informative comments out of $5 \mathrm{~K}$ on the four applications (the three which were used in the diary plus the added one). The ignored comments were mainly related to the technicality of the applications or adding no value to our analysis by being so generic, e.g. "I uninstalled this app, it exhausted the phone battery", "this is absolutely a nice app". 347 comments were made by male users, 254 by female users and 105 by users with undeclared gender.

Three main behaviour change theories guided the analysis of the selected applications; Control Theory [20], Goal-Setting Theory [21] and Social Cognitive Theory [22]. Control Theory suggests that the behaviour is regulated based on the person's intended behaviour seen as a goal. The control system will then compare the actual behaviour with that intended behaviour and actuate interventions if a deviation happens. Goal-Setting Theory emphasises the relationship between the goals and performance. Challenging goals appear to promote higher and persistent effort through motivating people to develop strategies that are more effective. The accomplishment will then reinforce further motivation due to individuals' satisfaction. Social Cognitive Theory suggests that behaviours are influenced by environmental aspects such as observing others. As such changing learning conditions can promote behavioural change. Overall, these theories were selected as they have been widely implemented in behaviour change research and had an evidence base [23]. In the data analysis phase, these established theories served as a conceptual basis for the priori coding approach to identify potential coding categories [17]. Hence, other theories have emerged during the process of the analysis such as Transtheoretical Model (TTM) 
[24]. Therefore, our methodology utilized behaviour change theories to explain the data analysed rather than controlling the analysis.

Qualitative content analysis was used. Although subjectivity is a common risk in this type of studies, the content analysis process included three researchers, two as evaluators and the third to take decision when a consensus was not reached.

\section{Results}

In this section, we will present the results of our analysis of the popular PIT, their features and how these features are seen by users and what concerns their usage would raise. The taxonomy which represents the results is shown in (Fig. 1). We concluded four main categories of features in this technology and we detail that in the next four subsections. This thematic map reflects the features that are considered by users as important. The PIT studied could also contain other features, which we omitted mainly because of the lack of relevance and influence from the users' perspective. We also elaborate on users' different views on the features.

\subsection{Monitoring}

Monitoring is an essential functionality of any self-regulation system. Measurement, comparison and the monitor are the core building blocks of the monitoring activity, while verifiability and transparency are monitoring-related principles that require a high degree of details to increase trustworthiness and reliability from a users' perspective especially for personal and behaviour-related information.

Measurement. Tracking time on-screen, i.e. duration, was the predominant method to calculate the addiction scores in all the reviewed applications. However, different applications rely on different metrics to measure time on screen. Users commented that these applications lack users' goals identification. As such, all types of usage are included in the measurement model without a special consideration of the intention and the reason for that usage. Time spent is perhaps not a meaningful measure for judging addiction if certain contextual factors are ignored and this requires intelligent and context-aware monitors that also look at the requirements and goals of the usage.

A time-based measurement model can be affected by the so-called passive usage. An example is the time between closing an application and screen auto-lock. Users commented that they would like not to have passive usage counted against them. On the other hand, receiving notifications, against their will including those coming from PIT, is a debatable case. Some commented that this would still be a type of usage as it requires additional cognitive load.

Frequency measurement is also used to estimate users' engagement with software products. The reviewed applications provided some frequency-based stats, e.g. screen unlocks. Calculating addiction scores is the quantification of a wide range of frequency-based and time-based stats to provide indications to the degree of usage. However, applying non-validated methods will lead to false conclusions.

The use of quantifying methods that are not validated will lead to false assertions. In more extreme bias users can use such misleading information to claim spontaneous recovery, which is a defensive mechanism known as the flight into health tactic [25]. 
For example, a user commented: "I did not ever know how often I checked my phone, I was using it about 200 times a day. Now I check it about 200 times a week thanks to much for curing me". Adopting factual and objective approaches, such as in the comment above, could provide more persuasive effect. Yet, careful feedback is still essential to avoid any misleading conclusions.

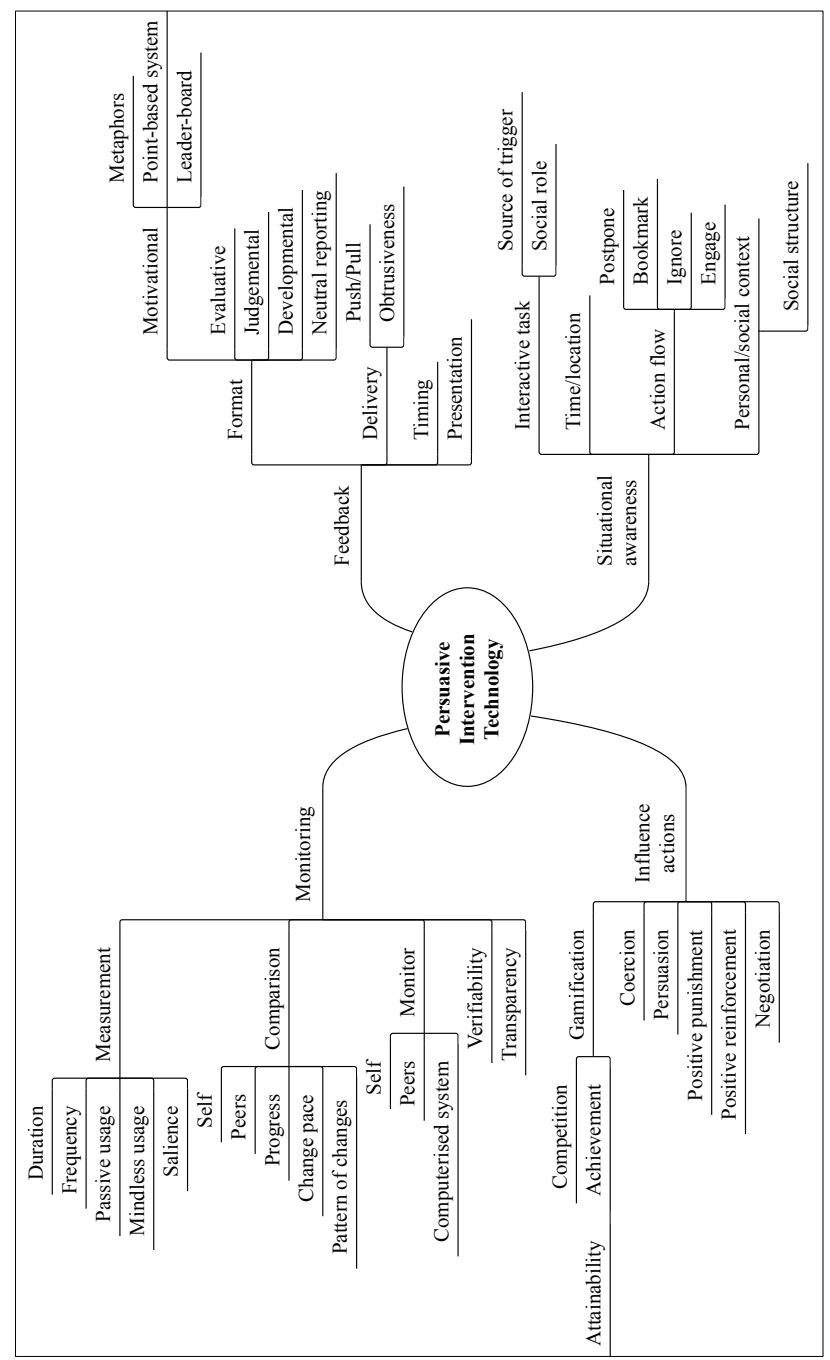

Fig. 1. Content analysis of users' views of PIT to combat digital addiction

Mindless usage is another factor that can also influence the measurement models. It can be characterised by the lack of conciseness and awareness during the present interaction with smartphones. This type of usage cannot be identified by duration or frequency. A technique like eye tracking might be able to identify this behaviour assuming that eye movements, which are guided by cognitive processes, during normal reading, are different from that during mindless reading and this helps automated 
detection.

Designing systems that can capture, measure and even intervene with the state of preoccupation, i.e. salience, is one of the open challenges for the design of interactive e-health and ICT-facilitate behaviour change. Salience attribute is one of the six clinical criteria proposed in [26] to identify addictive behaviours. It refers to the state when users are not actually engaged in the behaviour, yet they cannot stop thinking about it. For example, a user commented "I got so tired of thinking in Facebook statuses. Fellow addicts know what I am talking about". The challenge stems from the fact that such events do not occur in the system environment "points-based systems are a good motivational approach but how will [the system] monitor off-line behaviour or preoccupation". Thus, designers may need to incorporate users in the monitoring process and feedback loops in order to enable the system to react to such violation. However, proactive and intelligent masseurs that require stimulus identification and minimisation would be more advantageous from the usability perspective.

Comparison. All monitoring and feedback processes need to adopt some comparison approaches, or benchmarks, in order to measure users' progress. The overall findings indicate that users may need to be involved in the design phase to understand what would work well for them to avoid providing comparisons in a form that may negatively influence the user or cause them to disengage with the intervention system.

While the system can compare the user to his self-past, this can be done at different levels of granularity to reflect preferences on visualising the progress. For example, instead of comparing to the overall self-past usage, the system can compare the usage on each application individually. Such an approach was not implemented in all the reviewed applications. In fact, one of the applications provided very detailed stats but they were not in a quantified form to facilitate self-comparisons "I have not figured out yet how to see specifics of the applications that I do use ... just the overall usage!".

While comparing the performance with peers or self can help to motivate users progressive change, this social element may lead to negative experience, such as unhealthy competition. We argue that this approach can provide better outcomes when considering the stage of change according to the Transtheoretical Model (TTM) [24] stage of addiction, i.e. early, intermediate and severe and also the stage of treatment pre-, post- and during treatment. Also, the competition between peers is likely to impact self-esteem and self-efficacy (our perception of regulating the behaviour). A user who is in the early stages of the change, e.g. contemplation, may be compared to others in the advanced stages, e.g. maintenance. Although this upward social comparison is suggested to inspire those who are in worse off condition [27], it may also severely lower their self-esteem. For example, a user commented that gamifying the systems can be effective with caution "fun is needed but what about non-addicts appearing in a leaderboard?!". In other words, having users with different degrees of addiction in the comparison can have adverse effects. This also applies for those who are in the better off as they may use it as a defensive mean by using it and ignoring the other symptoms of addiction such as salience and conflict.

Showing the progress while performing the comparison can provide more meaningful information to users. This is a subtype of self-comparison by which users can compare themselves according to their own goals. It can also be a subtype of social- 
comparison when the goals setting is performed collaboratively as a group activity such as in surveillance systems.

The monitoring system can also compare the pace of users' progress toward the healthy usage. We labelled this as monitoring the changing pace. For example, one of the applications uses a metaphorical system in which users were enabled to grow trees to represent the progress towards healthier use. This application can be enhanced by monitoring the time between planting trees or how many trees planted in a given period of time to assess the pace of change. While planting trees entails implicit peers- or self-comparisons, the changing pace will be assessed by monitoring the outcomes. As such the reference point to compare with will keep changing in a progressive form.

As a follow-up, it may be argued that monitoring the pattern of changes can empower stage-based intervention systems. These systems are mainly built upon the TTM which takes pragmatic approach by focusing on "how" rather than "why" users progress through stages of change [24]. While there is still a lack of concrete evidence of its effectiveness on behavioural change [28], improving the tailoring system to take into consideration the unique motivational characteristics and trigging cues of each stage, provided promising outcomes [29]. The gap here is to identify the influential elements of the personalised information led to that improvement. This on its own is an indication of the applicability of stage-based interventions. However, one of the open challenges is collecting the evidence to confirm the stage transition to identify the current stage and then provide the right stage-matched interventions (SMI) [30]. In PIT, the monitorability of software-mediated interactions can help to facilitate monitoring that pattern of changes in order to assess the current stage and inform the tailoring system to provide effective and personalised interventions.

Monitor. This component refers to the agent who will do the monitoring activity itself. It can be performed by the users themselves, by peers or by a computerised system. Selecting one of those agents will have an influence on the other requirements and design choices. For example, the design of peer groups monitoring will require careful feedback engineering to avoid damage to social relationships or the development of maladaptive peer norms of usage. In groups' dynamics, the conformity effect can be a threat when a user temporary changes their behaviour only to conform and to avoid any contrary actions from peers. While this is considered a positive behaviour [31], this could be only positive in tasks adopted within a group to speed up achieving collective goals. In group-based treatment, however, this can be a threat as the relapse will be inevitable afterward, i.e. when users detach.

Verifiability. PIT need to provide means to verify the accuracy of their measurements and judgement of users' usage and behaviour in order to maximise their credibility and acceptability. Some of the reviewed applications provide detailed reports of usage. Such information can be used to support the claims of self-regulatory systems. One user criticised the subjectivity of the measurement of their addiction score or level: "The application allows seeing usage by time and some arbitrary addiction score". Our study indicated that the ability to verify the measurement process itself is much needed for such PIT especially if we consider the tendency of addicts to tolerate the increasing usage and deny reality. 
Transparency. Transparency has been highlighted as a key requirement. Users commented that they would like to see how scores are calculated and how the judgement on their usage is made. However, there was a wide range of scenarios that these systems utilize in the addiction scoring. Yet, users felt uncomfortable with not being involved in deciding them or even knowing them. This is a typical attitude in healthrelated interaction when patients require knowing details even if they may not fully understand them. One of the scenarios is the aforementioned "passive usage". Some of the passive usage cases were not identified by the designers. A user commented: "I didn't realise it counts the time the screen is left on even if you aren't using any of the applications". Thus, transparency requires carful elicitation and modelling to reach to an acceptable level. Participatory design and lifelong personalization approaches can support transparency by which users can be part of the decision-making process. This could increase adoption of the decisions and judgements made by the PIT but also introduce the risk of being biased and ineffective.

\subsection{Feedback}

Feedback is mainly to inform users about their performance and can take different forms. Feedback is one of the main pillars in self-regulation systems, which function to express users' status and to act as a motivational tool. The users' comments show that feedback techniques among these applications should be given a special considered in terms of the timing, format, delivery method and presentation.

Format. This refers to the type of content included in the feedback system. Feedback design can play a very important role to help different type of users to track their progress, yet should not conflict other design principles to avoid creating an addictive experience by itself. For example, a user criticised one of the applications "this application is addictive as well. Actually made me use my [mobile] more".

Motivational feedback is a complex type of feedback that visualises users' progress in a meaningful and gamified form to enrich their experience. Users commented that they would like to have point-based and metaphorical-based systems as well as leaderboards for this type of feedback "why can't I connect to Facebook? I wanted to compare the high scores". A user suggested that the metaphorical system could be improved by adding " a delay before the tree dies when using other applications. A notification could say, quickly, your tree is dying”. Leaderboards, however, could have a contradictory characteristic as they "may encourage unhealthy competition while they should be more about supporting each other not beating each other" especially in e-health solutions.

Evaluative feedback uses reference points to compare with, such as benchmarking, social or group's norms, but most importantly to show them how their performance scores were derived. Allowing users to set up their own reference points to compare with can be effective "I wish this application would allow me to set a time limit I feel is appropriate. If I use my phone for work, it is almost impossible to get [good] score which is pretty irritating when I have really cut down [time wasted] on my phone". The self-set reference point is linked to the concept of "goal choice" which is influenced heavily by past experience, past performance and some social influences [32]. A follow-up of this feedback is to guide the users to what areas they should improve 
in their usage and potentially enlighten them to think of what steps to take, e.g. using reductions and tunnelling [15]. This feedback needs to be timely to show the user the causes of the provided feedback when the usage contextual properties and cues are still fresh in their minds. However, users' feedback also indicated that timeliness might not be always appreciated especially if the user is still in a mental status of preoccupation about what they did or are doing on their smartphones.

Judgemental feedback can take an assessment manner in terms of judging the usage style to be right or wrong, healthy or addictive, etc. While this type can still carry some evaluative feedback attributes, it can be loaded with some emotive and judgmental terminologies, such as "you had an unusual and unhealthy usage style today" or "your usage is above the average time we expected for you today", etc. Users differ in terms of their motivations to accept and follow self-regulation systems. Sometimes this genuinely relates to their usage goals and other contextual factors, e.g. those who engage heavily with technology but still do not show addiction symptoms or those stating they are the "digital native" generation. As such, judgemental feedback messages may not suit all users "I just want data about how I use my phone, not silly platitudes about living my life to the fullest. This application was not for me". Techniques like authority and social proof [33] as well as basing the judgement on the measure or goal given by the user [21] would potentially help in increasing users' acceptance of such kind of feedback.

Developmental feedback can be used to offer fitting suggestions and tailored achievable plans, which can have greater persuasive powers. In terms of higher education, this maps to the formative assessment, which aims to constructively and iteratively evaluate performance and give suggestions for the next steps. A user commented that PIT would help them "to realise what they need to prioritise".

Some users preferred neutral reporting feedback, which only reports their usage stats without any further assessment and judgement "don't really like the score thing. Showing more real stats would be more useful".

Delivery. Self-regulation systems can communicate feedback messages following push or pull approaches. Designers need to understand users' requirements in terms of when to apply covert and overt feedback. The pull approach does not require the user to check their status as long as that will be prompted automatically following specific predefined event-based or interval-based modes. The push approach entails that the user is triggered to check their status. The pull approach can lead also to further addictive habits. For example, a user commented about an application uses trees metaphor "it seems counterintuitive to be building a forest on your phone, meaning you will inevitably keep coming back on to check your progress". The push approach, also, has a side effect by acting as a stimulus to initiate unnecessary usage "I love this application! But they should do something about getting notifications because those are tempting me". This approach may work for certain personality types and cultures. The pull approach could increase the sense of ownership and the fact that the user leads the querying process would encourage commitment and consistency [33] and hence the success of the change. We still do not have designated approaches for validating these design options when implemented in software systems.

Obtrusiveness can be, but not necessarily, one of the accompanying attributes when implementing the push approach, which may then affect users' experience. 
Obtrusive feedback, which can take a form of popup notifications, demands high attention and positions itself as a priority. Many users highlighted that feedback mechanisms were very obtrusive due to the lack of contextual considerations "this application doesn't let me define what works for me. Feels like a nosy parent ... there's a problem when a note pops up saying that I have spent too much time." However, as most interventions, obtrusiveness is still essential and participants stated that a "wake-up call" could be needed occasionally even if it violates some usability requirements.

Timing. The reviewed applications applied different usage-related timing strategies to deliver the feedback. Some were criticised of being very distractive, while others were very preferable. Feedback can be delivered during users' interaction with their mobile, after the usage (i.e. immediately after locking the screen or closing a specific application), while the user is away from the mobile, i.e. offline, or immediately after unlocking the screen. Users also commented that right timings are highly likely to motivate users. A user commented: "I like the fact that when I go to unlock my phone it tells me how many times I've unlocked it. Then I can think no I don't need to check".

Presentation. Presentation not only relates to the visual appearance of the feedback but also to what extent the information is consistent with users' attitudes and preferences, e.g. whether the message is a gain- or loss-framed, its friendliness, strictness, personal, etc. A user commented "there was a graph of how much I used my phone during the week. I found that quite useful because I could compare the days. Which ones I used it the most". In the systems that allow users to set their own plans, the colour coding can have a negative influence on how users set their own plans especially within social settings "If I am enabled to decide myself the maximum time I can use my phone, I am more likely to put high numbers. So in worst case, I'll get the orange colour. So I don't look that bad". As such, using statistical figures rather than colour coding in the intervention systems that enable self-setting of goals, can be more effective to eliminate self-bias. That bias can be used as a mechanism to minimize perceived impact on the self-image.

\subsection{Influence Actions}

This component aims at helping users regulating their usage by implementing behavioural change theories and techniques.

Punishment. Positive punishment can discourage behaviour by delivering a punishment when that behaviour is performed. Negative punishment, on the other hand, can discourage behaviour by removing positive stimulus when that behaviour is performed. Our study shows that these two forms of conditioning can strength likelihood of a healthier digital life style, e.g. "when I pick up my phone and get distracted, I get a notification telling that my tree died. This motivates me to stay focused next time". The tree is the symbolic object a user cares of and reduces the usage to avoid causing harm to it. 
Positive reinforcement. The system can be improved by implementing a rewarding scheme to assign specific rewards to different actions. Secondary actions linked to stimulus control such as a deliberate disabling Internet connection can be also rewarded "I wish you could get points for putting it in airplane mode or something".

Gamification. Self-regulation systems can be empowered by implementing some gaming elements to create a more engaging experience. Amongst the different game mechanics, competition and achievements seem to be predominant, still with potential for misuse.

Competition can be individual-based or even team-based to maximise users' experience. Any decline in the team performance can be perceived as an individual reasonability. Proper design of competition-based gamification can increase users' engagement significantly "I would suggest is if you added a 'buddy/friends list' so you can compete with your friends". The risk here is that the competition can take an adverse form, i.e. towards more use, or becomes itself addictive. For example, a user commented, "I can see making it competitive to worsen the addiction, would members want to get better and therefore get addicted to the points/rewards/making their avatar better?".

Providing users with tangible achievements can increase the likelihood of longterm engagement, which will particularly help to sustain users' behavioural change. Achievements are normally provided to users on an individual basis. However, users can be provided with individual achievement experience within the group context. As such, achievements can be provided to peers to gain social recognition. This is just an example to show how social and achievement aspects can be combined to create a very engaging experience, yet to be supported by consistency and commitment principles as a powerful social influence [33] to avoid relapse. A user commented: "I really like this application. Rather just sounding alarm or something, it gives a sense of accomplishment".

Some users criticised the rewarding system in some of the PIT. They pointed out that the long time and efforts needed to progress in the levelling system made it significantly difficult to get the rewards and this was very disappointing. On the other hand, the applications provide more attainable rewards seem to motivate users substantially "the little rewards or accomplishments I get are nice little reinforcements for low phone use". One approach that can be taken into consideration is to increase the difficulty as the user progress in the behavioural change stages, e.g. those of the Transtheoretical Model.

Coercion. The converge of the monitoring processes can have a significant impact on users' experience. We mean by coverage what can be included in the monitoring, e.g. application usage, lunches, device unlocks or even within-application interactions such as likes, posts and sharing. For example, some of the reviewed applications provided functionality to exclude applications from the monitoring process or to allow user specify monitoring specify monitoring preferences "there should be a new feature in which the phone will close on its own after a certain time period which can be set by the users". Such flexibility is required to avoid unnecessary coercive interventions. Users normally have the tendency to exclude work-related applications such as email clients and navigation applications. However, this flexibility would certainly 
need to be implemented with high caution, as addicts tend to deny reality and invent untrue reasons for excluding an application "Some people like me need to not be able to manually [stop the monitoring]". Other applications provide "snoozing" feature to support task continuity or even to pause the monitoring activity "a pause feature would be amazing because sometimes I want to get food while studying and I don't want to spend time on the app".

Persuasion. Persuasion is a very important principle to influence users' intentions and behaviours. Tunnelling, social comparison, reminding, rewarding and suggestion were the most requested techniques by the users "it would be better to get software recommendations for planning the allowed time of usage and to update this based on my actual usage". Research on evaluating effectiveness and sustainability of the technology-assisted version of such techniques in general, and for DA in particular, is still to be done. For example, personality traits besides the type and stage of addiction could have a high impact on the acceptance and effectiveness of persuasion and also coercion.

Negotiation. Users' conflicting requirements require carful identification and resolution. The question here is how to intelligently negotiate requirements in a way that considers the peculiarities in addicts' behaviour such as tolerance and denial of reality. For example, most of the PIT enable users to exclude certain applications from the monitoring activity and this was perceived as a desirable functionality "I wish this application would allow you to set a time limit you feel is appropriate for green, or have certain applications like e-mails and phone calls do not count against you”. We argue, here, that it would be more efficient to exclude them in the influence layer, but not monitoring and feedback according to (Fig. 1). This is to alert users when addiction patterns are identified in one of the excluded applications. In some scenarios, however, coercive approaches can be used when such patterns are detected as these systems should perceive users as two interconnected personas; current user and userto-be.

\subsection{Situational awareness}

Situation includes a wide range of variables related to the performed task. The lack of knowledge about tasks' context as well as poor elicitation of a user's mental models, can affect user experience when implementing self-regulation systems. Thus, expanding the exploratory investigation to include contextual factors is essential to provide empirical rational needed to inform the design of software-based interventions and promote the intended behavioural change. Data analysis of the collected comments highlighted the critical principles below.

Interactive task. Users highlighted that the system should distinguish between tasks in terms of their nature, e.g. seriousness "I just uninstalled this after I nearly had an accident. Upon setting GPS map route the reminder pop out blocking my map in the midst of driving", another commented "fails to meaningfully distinguish between productive phone use and addiction", and also who initiated it, i.e. triggered by attribute "only counts the interactions initiated by the phone user. If a call comes in, it should 
not be counted". Again, there seems to be a grey area between the two cases, e.g. receiving a message on Facebook as a result of sharing a post and the escalating commitment on social networks. Here, we propose the severity as an important quantifiable task-related attribute to enrich measurements models. In order to achieve this, different interactions need to be categorised based on their implications on the usage style. In the previous example, the sharing a post is likely to cause a high volume of responses, which can aggravate habitual checking. This is unlike other tasks which can be categorised as human to machine interactions. Such interactions can be less problematic as the social element is messing. This also suggests categorising interactions based on their social roles which denote the notion of the extent to which interaction motivate or demotivate face-to-face interactions. For example, interactions that encourage face-to-face communications, such as organising events using softwaremediated tools, may need to be treated as positive interactions that should be promoted by the system rather than those encouraging online participation which can still be counted against addiction score. Thus, understanding the goal of the interactions and the task being done is essential for decision-making, e.g. on the type of feedback to give and measurement to apply.

Time/Location. The system should enable users to decide when and where they want to be monitored. These contextual variables can be very sensitive when it comes to feedback messages. Time, location and tasks can also be combined to identify problematic usage. For example, users can be enabled to select the morning as a working period and any Facebook usage during that time whether it is exempted from monitoring or not will be counted in the addiction score. However, implementing such scenario for users who do not want coercion approaches can create conflicting requirements.

Action flow. In less coercive settings, the design of PIT is required to minimise affecting user experience. One way of doing that is by providing users with more flexibility to support taking appropriate decisions as intuitively as possible. Research has shown that self-control has very limited resources for tasks involving a strong desire. So, when users utilise the power of self-control in the initial task, subsequent tasks are compromised due to "self-control depletion" [23]. A user commented "It needs a strong mechanism to prevent us from simply turning off [digital addiction] rules. This is because self-control is a limited resource that depletes as the day goes by. So when it's late in the afternoon won't have the energy to stop myself from simply disabling the rules". As such, the software must use up this valuable resource intelligently to avoid "ego depletion". One way of doing that through intervention systems is to use self-control resources for the high problematic tasks only such as entirely blocking certain applications. Bolstering self-control through software means is an important aspect to promote behavioural change. For this, we propose the postponing and bookmarking techniques to supports task continuity for users who do not like strict coercive approaches. The former technique enables the user to postpone a promoted desired task to be performed later but at the right time. As such the spontaneous urge to perform the task will be controlled with minimum use of self-control resources since the task can be performed later. The bookmarking technique is to maintain the point of usage before the intervention happened. A user commented: "the application will not kick me out when time is up. However, it will prevent me from starting it 
again if I have used it already for longer than the allowed time". While both techniques could be particularly the case with gaming addicts, implementing such interaction is irrelevant to multiplayer video games where more than one player engaged in the same game simultaneously. This highlights the need for consideration of conflicting requirements, which can be addressed by an ontology supported by behavioural change theories and domain reasoner to help designers mapping the interaction artefacts to the application domain.

The intervention software can prompt all muted notifications or those were postponed during the controlled time. One way of strengthening self-efficacy is by utilising the actions taken towards these notifications. Simply by counting the ignored ones for the user not against him and to reflect that positively on his addiction score. As users are still expected to engage with those notifications, they should not be penalised when that is performed out of the controlled time. This emphasises the importance of having considerate interventions which can be categorised as a special form of considerate requirements for social software proposed in [34]. For example, a user commented, "I don't look at my phone when I drive so it would be nice to [reward me]". This class of requirements seems to be fundamental and should be advocated to allow evaluating such interactions against addicts' perception of consideration to avoid any potential harms resulting from interventions.

Personal/social context. Personal context relates to the innate feeling and status of the user, e.g. mood. Social context refers to the both the position of the user within a group either in the real world or on a social network. Sensitivity to such context is hard to achieve but with advances in sensing mechanisms, e.g., smart watch, and machine intelligence, we speculate this would become eventually a reality. Social elements can influence users' perceptions towards intervention mechanisms. Yet, what is accepted and being effective in human-to-machine interactions, might be harming in social settings due to different factors such as digital identity. We looked at how social context would affect users' willingness to use this type of intervention systems. User raised the importance of having a space that is free of criticism "I think it needs to be a safe space that people can feel free to explore their issues without fear of criticism". Having the social elements would also influence what feedback format should be adopted. For example, judgemental feedback is not preferable in such settings " $I$ wouldn't consider any group which labelled an individual's use of a medium or set of media in such a sweepingly judgemental way to be an efficient mode of help". In terms of being within an online social network, users also raised the need for considering the social structure within social intervention system. A user commented, "I prefer groups in which members know each other. Nothing is against family members being in the group. But they might be still seen as strangers by others and this may influence how they communicate with me, e.g. when my daughter is in the group".

\section{Designing PIT to Combat Digital Addiction}

Our analysis in section 3 demonstrated the need for careful considerations and design principles when using PIT in the domain of DA. In this section, we discuss those aspects in light of the literature and other relevant study and then highlight the need for testing and validating methods for this technology. Finally, we pinpoint the main 
issues and challenges in designing PIT for DA and where the future research studies are needed.

PIT is an example of how technology is enabling individuals to engage with the field of behaviour change in a way that has in the past primarily been restricted to health educators and policy makers. Researchers and practitioners working in behaviour change have developed an extensive research literature on theories of behaviour change, and an evidence base to support the efficacy of different techniques. This knowledge is reflected in sources such as NICE (National Institute for Health and Care Excellence) guidelines on behaviour change for individuals (https://www.nice.org.uk/Guidance/PH49), which advise on best practice. It is interesting how many of the characteristics of the selected PIT mirror the NICE recommendations for behaviour change in other potentially addictive behaviours such as alcohol and tobacco use. For example, as noted, all of the applications include some form of monitoring, which is the first step of many behaviour change approaches in alcohol and drug use.

Nevertheless, behaviour is determined by a multitude of factors, and as such, there can be a discrepancy between the behaviour change strategies, which should be expected to work according to theory and those which have an actual impact. PIT may or may not have some basis in behaviour change theory, but even if designed with the best of intentions and some relevant knowledge it may not provide any benefit to users, and may even have harmful effects. There are several examples of large-scale behaviour change campaigns that have been unsuccessful, such as the DARE (Drug Abuse Resistance Education) programme in the USA that failed to bring about change and was alleged to inadvertently reduce the self-esteem of participants [35].

Even simple and apparently commonsensical strategies such as suggesting that the individual avoids thinking about certain behaviour may be harmful. For example, it has been noted that advising people to try and avoid thinking about certain behaviour, as often done for instance in relation to smokers and avoiding thinking about cigarettes, can actually increase the compulsion to engage in that behaviour [17]. Care must also be taken that a behaviour change strategy is not chosen simply because it is opportune. PIT is especially suited to social comparisons that allow users to see how their usage compares to that of their peers, with the assumption being that those who behave in an excessive way will reduce their usage. However as noted with regards to alcohol use in American college students individuals may base their identity of being the most extreme amongst their group, in which case highlighting to them how they compare to their peers may on reinforce that behaviour [36]. Finally, in any behaviour change, there is the issue of reactance. This refers to when individuals feel that they are being manipulated and respond by engaging more actively in the behaviour that they feel they are being dissuaded from. Overall it could be argued that behaviour change is easy to achieve, but ensuring that the change occurs in the intended direction is much more challenging.

We can conclude that the requirements engineering and design for PIT introduce challenges in several areas including the decision on the relevant stakeholders and their decision rights and priorities. The failure stories of traditional behaviour change practices send also an alarm on the need for novel testing and validation for PIT. Testing for long-term consequences, e.g. decreased self-esteem, and collective side effects, e.g. creating certain norms of usage, would necessitate novel ways on validating 
whether such software does meet the requirements sustainably and without unpredictable side effects. This requires a joint effort of multiple disciplines including requirements engineering, human-computer interaction and psychology.

The term universal design describes the concepts of designing for all regardless of their age, gender and abilities [37]. As such, PIT should not be designed with the mind-set of one size fits all and should cater for complex inter-related networks of variables. We view the domain of behavioural change, as important effort to provide reactive approaches to deal with this issue. However, there is an evident lack of test frameworks to validate the effectiveness of intervention systems built based on the theories of behavioural change. Validating the effectiveness of such technology requires a unique set of pre-conditions such as willingness to change, openness to shortcomings, being free from denial of reality and also the seriousness of the condition. The challenge here is how to measure these factors, e.g. change readiness, to control their influence on the validity of the intervention system.

Also turning the system into social software by including peers in the monitoring activity requires assessing the long-term outcomes and their sustainability. The validity of such change might be distorted due to various confounding factors arising from peer pressure and other negative influences such as the short-term change only to conform to the group's norms. Table 2 summarizes the findings of the paper from the perspective of users' experience (UX) and what could be the source of concerns from the design perspective and also psychological and contextual perspectives.

Table 2. Design concerns and their potential sources in PIT to combat DA

\begin{tabular}{|c|c|}
\hline UX concerns & Source of concerns \\
\hline Lack of interest & $\begin{array}{r}\text { Experience fails to engage, ineffective rewarding system, poor levelling } \\
\text { design, willingness and readiness to change }\end{array}$ \\
\hline Lack of trust & $\begin{array}{r}\text { Unreliable addiction scoring, lack of verifiability and transparency, } \\
\text { uncertainty of agenda of application's developer(s) }\end{array}$ \\
\hline Lowering self-esteem & $\begin{array}{r}\text { Peer-pressure, upward social comparisons, low sense of self-efficacy, } \\
\text { assigning to non-matched groups }\end{array}$ \\
\hline $\begin{array}{c}\text { Creating } \\
\text { misconceptions }\end{array}$ & $\begin{array}{c}\text { Addiction scoring, minimising the seriousness of the addicting, } \\
\text { providing non-stage matched interventions }\end{array}$ \\
\hline $\begin{array}{c}\text { Biased decisions } \\
\text { Creating addictive } \\
\text { experience }\end{array}$ & $\begin{array}{r}\text { Downward social comparisons, self-set goals, flight into health, denial } \\
\text { of reality, influence from past experience and performance }\end{array}$ \\
\hline $\begin{array}{c}\text { Impacting user } \\
\text { experience }\end{array}$ & $\begin{array}{c}\text { Obtrusiveness, distraction, coercive techniques, affecting workflow, } \\
\text { lack of requirements negotiations, neglect personalised experience }\end{array}$ \\
\hline Unsustainable change & Social elements (e.g. conformity effect), losing interest \\
\hline Self-image impact & Identification as addict, experience of relapsing \\
\hline
\end{tabular}

\section{$5 \quad$ Study Limitations}

The study has two main limitations that may have an influence on how the features in PIT were seen by the participants. Firstly, in the measurement of participants' level of 
addiction, we used the CAGE questionnaire as a simple and inexpensive instrument that does not cater for the wide spectrum of cases and levels of addiction. Generally, psychometric measures have major issues in the addiction criteria itself such as the lack of considering the context of use, the aspects related to the temporal dimension such as compensating relationship breakdown [38] and preoccupation which has been highlighted in the results section of this paper. This explains why "no gold standard" for diagnosing and assessing DA yet exist [39]. The second limitation relates to the fact that those who installed the apps were help-seekers only. Apparently, non-help seekers may have different views and perception about features in PIT. Finally, an additional limitation is about the choice of the apps. Analysing extra apps might lead to discovering additional concepts and risks of this technology in the domain of DA.

\section{Conclusions and Future Work}

In this paper, we explored users' perception of PIT for combating DA and argued the need for a more careful and holistic approach to technology-assisted behaviour change in DA. The unique contribution of this work derives from its attempt to analyse various views, potentials and risks related to a dual use and dual effect of such technology.

Throughout the analysis of the users' comments and the developers' feedback to those comments on the online forum, we noticed the rush to embrace this technology in order to cope with the market demand without careful consideration of its adverse effects. A prominent example is dealing with fundamental issues such as the measurement of DA, which requires extensive research, as merely a technical problem promised to be addressed in the next updates. Hence, the outcome of technology designed for behavioural change is currently doubtful at least in the area of DA. We argue that more research is needed in the area of testing and validating the effectiveness of this technology on the intended behaviour in the short and long terms. For example, how can we assess the threats of users' rejection of the interactive intervention systems? Another example is the negative feelings that can be evoked, such as guilt and obligation, of certain design elements. The former may reinforce the relapse behaviours and the latter may aggravate addiction-related behaviours such as fear of missing out. While trade-offs is a common observation in HCI research, in the domain of addictive behaviours such compensation may propose undesirable effects.

The participatory approach can help to reduce unpredictable effects. However, there is a need to devise methods and guidelines supported by best practices to govern and engineer the users' involvement itself especially for the digital addicts user groups who may exhibit a denial of reality. Hence, more research is still needed to utilize user-centred and participatory approaches for designing PIT to combat DA. For example, it is not clear whether and how to involve ex-addicts in the design and test processes. While ex-addicts may have more empathy for addicted users, they might dictate their opinion due to their bias and their own experience.

In our future work, we will define metrics for addictive software and addictive behaviour to make DA subject to a more accurate monitoring and adjustment process. We will study the design of software-based behaviour change at the precautionary and recovery stages. In particular, we will focus on motivational approaches and the use of their software-based version, for example, persuasive technology and entertainment 
computing [40]. We aim to investigate the requirements engineering and software validation for DA-related behavioural change and their challenges, for example the denial of requirements of addicts and their conflicts. We will investigate the stakeholders set and their decisions rights in the engineering process, including addicts, the ethical issues around the engineering process and the sustainability of softwarefacilitated prevention and early-intervention for DA and their potential short and longterm side-effects. This will obviously require an inter-disciplinary research.

Acknowledgements. This research has been partially supported by Bournemouth University through the Fusion Investment Fund and PGR Development Fund and also by StreetScene Addiction Recovery. We would like also to thank Yasmeen Abdalla for her valuable contribution in conducting the diary study in the early stages of this research.

\section{References}

1. Kuss, D.J., Griffiths, M.D.: Online Social Networking and Addiction-A Review of the Psychological Literature. International Journal of Environmental Research and Public Health 2011, Vol. 8, Pages 3528-3552. 8, 3528-3552 (2011).

2. Echeburúa, E., de Corral, P.: Addiction to new technologies and to online social networking in young people: A new challenge. Adicciones. (2009).

3. Young, K.S.: Internet addiction: symptoms, evaluation and treatment. Innovations in clinical practice: A source book. (1999).

4. Young, K.S., de Abreu, C.N.: Internet Addiction: A Handbook and Guide to Evaluation and Treatment. (2011).

5. Kuss, D.J., Griffiths, M.D., Binder, J.F.: Internet addiction in students: Prevalence and risk factors. Computers in Human Behavior. 29, 959-966 (2013).

6. Ali, R., Jiang, N., Phalp, K., Muir, S., McAlaney, J.: The Emerging Requirement for Digital Addiction Labels. REFSQ. 9013, 198-213 (2015).

7. Alrobai, A., Phalp, K., Ali, R.: Digital Addiction: A Requirements Engineering Perspective. Requirements Engineering: Foundation for Software Quality. 8396, 112-118 (2014).

8. Bewick, B.M., Trusler, K., Mulhern, B., Barkham, M., Hill, A.J.: The feasibility and effectiveness of a web-based personalised feedback and social norms alcohol intervention in UK university students: A randomised control trial. Addictive Behaviors. 33, 1192-1198 (2008).

9. Bricker, J.B., Mull, K.E., Kientz, J.A., Vilardaga, R., Mercer, L.D., Akioka, K.J., Heffner, J.L.: Randomized, controlled pilot trial of a smartphone app for smoking cessation using acceptance and commitment therapy. Drug and Alcohol Dependence. 143, 87-94 (2014).

10. Dayer, L., Heldenbrand, S., Anderson, P., Gubbins, P.O., Martin, B.C.: Smartphone medication adherence apps: Potential benefits to patients and providers. J Am Pharm Assoc (2003). 53, 172-11 (2013).

11. Pagoto, S., Schneider, K., Jojic, M., DeBiasse, M., Mann, D.: Evidence-Based Strategies in Weight-Loss Mobile Apps. American Journal of Preventive Medicine. 45, 576-582 (2013).

12. Dennison, L., Morrison, L., Conway, G.: Opportunities and challenges for smartphone applications in supporting health behavior change: qualitative study. Journal of medical .... 15, e86 (2013).

13. Dumas, B., Signer, B., Lalanne, D.: Fusion in multimodal interactive systems: an HMMbased algorithm for user-induced adaptation. EICS. 15-24 (2012).

14. Sutcliffe, A.G., Fickas, S., Sohlberg, M.M.: Personal and Contextual Requirements Engineering. RE. 19-30 (2005).

15. Fogg, B.J.: Persuasive Technology: Using Computers to Change What We Think and Do 
(Interactive Technologies). (2002).

16. Torning, K., Oinas-Kukkonen, H.: Persuasive system design: state of the art and future directions. PERSUASIVE. 30 (2009).

17. Lazar, D.J., Feng, D.J.H., Hochheiser, D.H.: Research Methods in Human-Computer Interaction. John Wiley \& Sons (2010).

18. Widyanto, L., Griffiths, M.D., Brunsden, V.: A Psychometric Comparison of the Internet Addiction Test, the Internet-Related Problem Scale, and Self-Diagnosis. Cyberpsychology, Behavior, and Social Networking. 14, 141-149 (2011).

19. Ewing, J.A.: Detecting alcoholism: the CAGE questionnaire. Jama. (1984).

20. Carver, C.S., Scheier, M.F.: Control theory: A useful conceptual framework for personality-social, clinical, and health psychology. Psychological Bulletin. 92, 111-135 (1982).

21. Locke, E.A., Latham, G.P.: A theory of goal setting \& task performance. (1990).

22. Bandura, A.: Social cognitive theory: An agentic perspective. Annu. Rev. Psychol. 52, 1$26(2001)$

23. Webb, T.L., Sniehotta, F.F., Michie, S.: Using theories of behaviour change to inform interventions for addictive behaviours. Addiction. 105, 1879-1892 (2010).

24. Prochaska, D.J.O.: Transtheoretical Model of Behavior Change. In: Gellman, M.D. and Turner, J.R. (eds.) Encyclopedia of Behavioral Medicine. pp. 1997-2000. Springer New York, New York, NY (2013).

25. Frick, W.B.: Flight into health: A new interpretation. Journal of humanistic psychology. (1999).

26. Griffiths, M.: A "components" model of addiction within a biopsychosocial framework. J Subst Use. 10, 191-197 (2005).

27. Taylor, S.E., Lobel, M.: Social comparison activity under threat: Downward evaluation and upward contacts. Psychological Review. 96, 569-575 (1989).

28. West, R.: Time for a change: putting the Transtheoretical (Stages of Change) Model to rest. Addiction. 100, 1036-1039 (2005).

29. Borland, R., Balmford, J., Hunt, D.: The effectiveness of personally tailored computergenerated advice letters for smoking cessation. Addiction. (2004).

30. Sutton, S.: Back to the drawing board? A review of applications of the transtheoretical model to substance use. Addiction. (2001).

31. Toseland, R.W., Rivas, R.F.: An Introduction to Group Work Practice. Pearson Education, Limited (2005).

32. Locke, E.A., Latham, G.P.: New Directions in Goal-Setting Theory. Current Directions in Psychol Sci. 15, 265-268 (2006).

33. Cialdini, R.B.: Influence. HarperCollins (2009)

34. Ali, R., Jiang, N., Jeary, S., Phalp, K.: Consideration in software-mediated social interaction. RCIS. 1-11 (2014).

35. Lynam, D.R., Milich, R., Zimmerman, R., Novak, S.P., Logan, T.K., Martin, C., Leukefeld, C., Clayton, R.: Project DARE: No effects at 10-year follow-up. Journal of Consulting and Clinical Psychology. 67, 590-593 (1999).

36. Carter, C.A., Kahnweiler, W.M.: The efficacy of the social norms approach to substance abuse prevention applied to fraternity men. Journal of American College Health. 49, 66-71 (2000).

37. Center for Universal Design: The Principles of Universal Design, https://www.ncsu.edu/ncsu/design/cud/about_ud/docs/use_guidelines.pdf.

38. Griffiths, M.: Internet Addiction - Time to be Taken Seriously? Addiction Research. 8, 413-418 (2000)

39. Kuss, D.J., Griffiths, M.D., Karila, L., Billieux, J.: Internet addiction: a systematic review of epidemiological research for the last decade. Curr. Pharm. Des. 20, 4026-4052 (2014).

40. Jiang, J., Phalp, K., Ali, R.: Digital Addiction: Gamification for Precautionary and Recovery Requirements. REFSQ Workshops. 224-225 (2015). 